\title{
CORRELAÇÃO ENTRE PRESSÃO ARTERIAL E PESO EM CRIANÇAS E ADOLESCENTES DE UMA ESCOLA MUNICIPAL DO NOROESTE PAULISTA
}

\author{
Correlation between blood pressure and weight in \\ children and teenagers from a municipal school in the \\ northwestern region of São Paulo \\ Correlación entre presión arterial y peso en niños y adolescentes
de una escuela municipal del Estado de São Paulo
}

\section{RESUMO}

Verificar a correlação entre alterações de peso e pressão arterial em escolares do ensino fundamental de uma escola municipal do noroeste paulista. Método: Estudo quantitativo, descritivo e correlacional. Após a coleta e categorização do peso e pressão arterial de 148 escolares, os dados foram digitados no Excel e analisados no programa estatístico Statistical Package for Social Science, versão 12, utilizando-se estatística descritiva e inferencial (tabela de contingência e teste de correlação de Pearson). Resultados: 0 sexo predominante foi masculino (63,5\%), e a idade média 8,4 anos; 36,5\% apresentaram índice de massa corpórea alterado (3,4\% baixo, 10,1\% sobrepeso e 23\% obesidade); 4,7\% apresentaram pressão arterial limítrofe, e 9,5\%, hipertensão. 0 teste de correlação indicou correlação forte e positiva entre peso/índice de massa corpórea e pressão arterial. Conclusões: Os resultados contribuem para o diagnóstico precoce de alterações pressóricas na população estudada e sua prevenção nos indivíduos obesos e com sobrepeso.

Palavras-chave: Saúde da Criança. Hipertensão. Sobrepeso.

\begin{abstract}
To verify the correlation between weight and blood pressure alterations on students from a public basic education school from the interior of São Paulo. Method: Descriptive and correlational study, with quantitative approach. After collected and categorized the weight and blood pressure of 148 students, the data were typed in Excel and analyzed, through descriptive and inferential stats (cross-tabs and Pearson's correlation test), using the program Statistical Package for Social Science $12^{\text {th }}$ version. Results: The prevailing gender was male (63.5\%) and the average age was $8.4 ; 36.5 \%$ presented altered corporal weight (3.4\% low weight, $10.1 \%$ overweight and $23 \%$ obesity); $4.7 \%$ presented bordering levels of blood pressure and $9.5 \%$ presented hypertension. The correlation test indicated strong positive correlation between weight/corporal weight and blood pressure. Conclusions: The results contribute to the precocious diagnosis of blood pressure alterations in the researched population as well as to its prevention in obese and overweight individuals.

Keywords: Child health. Hypertension. Overweight.
\end{abstract}

\section{Resumen}

Evaluar la correlación entre los cambios de peso y la presión arterial en niños que cursan la enseñanza primaria de una escuela municipal en el noroeste del Estado de San Paulo. Método: Estudio cuantitativo, descriptivo y correlacional. Después de identificar y clasificar el peso y la presión arterial de 148 estudiantes, los datos fueron digitados para Excel y analizados en el programa estadístico Statistical Package for Social Science, versión 12, utilizandose estadística descriptiva e inferencial (tabla de contingencia y el coeficiente de correlación de Pearson). Resultados: El sexo predominante fue el masculino (63.5\%) y la edad promedio, 8.4 años; $36.5 \%$ presentaron un índice de masa corporal con alteraciones (3.4\% bajo, $10.1 \%$ presentan sobrepeso y $23 \%$ presentan obesidad), $4.7 \%$ presentaron cifras limítrofe de hipertensión arterial y 9.5\% presentaron hipertensión. el coeficiente de correlación indicó una correlación fuerte y positiva entre el peso/índice de masa corporal y la presión arterial. Conclusiones: Los resultados contribuyeron al diagnóstico precoz de los cambios en la presión de la población estudiada y su prevención en individuos obesos y con sobrepeso.

Palabras clave: Salud del niño. Hipertensión. Sobrepeso.

${ }^{1}$ Enfermeira. Mestranda do Programa de Pós-Graduação em Enfermagem da Universidade Federal de São Carlos. Professora Assistente do Departamento de Enfermagem da Universidade Paulista - UNIP, campus São José do Rio Preto - SP. Brasil. E-mail: ma.zanoti@yahoo.com.br, ${ }^{2}$ Enfermeira. Mestre em Saúde Pública. Especialista em Laboratório do Departamento de Enfermagem Materno-Infantil e Saúde Pública da Escola de Enfermagem de Ribeirão Preto - Universidade de São Paulo. Brasil. E-mail: jcoelho@eerp.usp.br, ${ }^{3}$ Enfermeira. Doutoranda do Programa de Pós-Graduação Enfermagem Fundamental da Escola de Enfermagem de Ribeirão Preto - Universidade de São Paulo. Brasil. E-mail: marcelamanetti@usp.br 
Correlação pressão arterial e peso em crianças Zanoti MDU, Pina JC, Manetti ML

\section{INTRODUCÃO}

A obesidade exógena é considerada um problema de saúde pública, uma vez que este distúrbio nutricional está em ascensão no mundo moderno. Tal desequilíbrio energético é facilmente observado em crianças, pois estas, atualmente, apresentam um comportamento sedentário, tendo como atividades lúdicas e de lazer passar muito tempo em frente à televisão, computadores e videogames, reduzindo a prática de atividade física e mantendo um predominante consumo de alimentos com alta densidade energética. ${ }^{1-2}$

Os hábitos alimentares inadequados, a obesidade dos pais, a inatividade física e o desmame precoce são fatores que podem predispor a criança ao excesso de peso. Assim, a família tem grande influência na gênese da obesidade, uma vez que seus estilos de vida sedentários e hábitos alimentares inadequados influenciam o estilo de vida adotado pela criança. ${ }^{2}$

Em estudo que buscou avaliar o estado nutricional e a associação com o padrão de atividade física em escolares da Rede Municipal de Ensino de Corumbá (MS), os dados evidenciaram a importância em promover mudanças no estilo de vida com a adoção de hábitos saudáveis, desde a infância, e a sua manutenção por toda a vida, uma vez que crianças ativas favorecem uma população adulta também ativa e saudável contribuindo, consequentemente, para a redução da incidência de morbidade e mortalidade na idade adulta. ${ }^{3}$

Considerada uma pandemia, a obesidade infantil apresenta custos socioeconômicos significativos por sua elevada morbimortalidade, além de predispor o indivíduo a doenças que poderão reduzir sua expectativa e qualidade de vida. ${ }^{4}$ Nesse sentido, as crianças obesas têm maior probabilidade de apresentar dislipidemias, hipertensão e diabetes, entre outras patologias. $^{3}$

A hipertensão arterial (HA) é considerada o primeiro fator de risco para as doenças cardiovasculares, as quais constituem a principal causa de morte no Brasil. ${ }^{5} \mathrm{~A}$ literatura nacional $\mathrm{e}$ internacional refere uma ampla variação na prevalência da $\mathrm{HA}$ infantil, demonstrando valores entre $1 \%$ e $13 \%$, a depender da metodologia empregada. No entanto, recentes estudos epidemiológicos brasileiros têm demonstrado prevalência entre $6 \%$ e $8 \%$ na população infantil. ${ }^{6}$

De acordo com um estudo desenvolvido na Bahia ${ }^{3}$, a presença de sobrepeso, a obesidade e o fato de estudar em escola privada são considerados fatores preditivos independentes para a $\mathrm{HA}$, sugerindo um papel importante de fatores biológicos e ambientais na gênese da $\mathrm{HA}$ em crianças. No entanto, os autores ressaltam que a associação entre $\mathrm{HAe}$ o fato de estudar em escola privada contrariam dados epidemiológicos clássicos na população adulta, que
Esc Anna Nery Rev Enferm 2009 out-dez; 13 (4): 879-85

demonstram uma maior prevalência desse distúrbio entre classes sociais menos favorecidas.

A literatura atual preconiza a aferição da pressão arterial como parte do exame físico de crianças a partir de três anos de idade, podendo ser realizada antes dessa idade, rotineiramente, para diagnosticar precocemente doenças potencialmente graves, como a doença renovascular.?

É crescente o número de crianças e adolescentes com hipertensão arterial, e, por isso, medidas preventivas são necessárias para minimizar os riscos cardiovasculares na vida adulta. $^{8-9}$

Devido à pouca atenção e ao despreparo das equipes de saúde que tratam de crianças, a hipertensão, que pode ser minimizada ou até curada se for diagnosticada precocemente, acaba por se instalar e perdurar nesses pacientes. ${ }^{10}$ Contudo, além do preparo das equipes técnicas, a prevenção, o diagnóstico e o tratamento precoce da $\mathrm{HA}$ envolvem o esclarecimento de educadores e familiares sobre a importância de mudanças no estilo de vida. Desse modo, as crianças não somente se beneficiariam da prevenção e do tratamento da HA, mas se preveniriam de todas as comorbidades associadas ao sobrepeso eà obesidade. ${ }^{4}$

Em relação à enfermagem, a assistência prestada desde a atenção básica, envolvendo ações de educação em saúde e diagnóstico precoce, contribui para resultados relevantes no histórico dessas crianças e adolescentes com obesidade e hipertensão arterial, mudando, com orientações e acompanhamento, o presente e futuro desses clientes.

Considerando a importância do diagnóstico precoce da $\mathrm{HA}$ infantil, o presente estudo tem como objetivo geral verificar a correlação entre alterações de peso e pressão arterial em escolares do ensino fundamental de uma escola pública em um município do interior paulista. Tem como objetivos específicos verificar a prevalência de sobrepeso e obesidade, identificar a ocorrência de alterações na pressão arterial e verificar a influência do peso nos níveis pressóricos entre as crianças estudadas.

\section{METODOLOGIA}

Estudo transversal, descritivo e correlacional, com abordagem quantitativa dos dados, realizado em uma escola da rede pública do município de São José do Rio Preto - SP.

0 projeto de pesquisa foi aprovado pelo Comitê de Ética e Pesquisa do Instituto do Coração de Rio Preto/INCOR Rio Preto em 20/08/2008, sob o protocolo 0014.0.137.000-08.

A população de estudo foi composta por 148 escolares, na faixa etária de 6 a 11 anos, regularmente matriculados na referida escola pública de ensino fundamental. 
Os dados foram coletados em junho de 2008, a partir de um instrumento desenvolvido pelas pesquisadoras, obtendose os seguintes dados: sexo, idade, peso, altura e pressão arterial.

As crianças e adolescentes ${ }^{1}$ foram pesados descalços $\mathrm{e}$ com roupas leves; foi pedido para retirarem chaves, óculos, cintos, telefone celular e quaisquer outros objetos que pudessem interferir no peso total. Utilizou-se balança mecânica de plataforma, certificando-se de que estivesse afastada da parede. Após a calibração da balança, esta foi travada e só então a criança, ou adolescente, subiu na plataforma para ser pesada, posicionando-se de costas para a balança, no centro do equipamento, ereta, com os pés juntos e os braços estendidos ao longo do corpo, mantendo-o parado nessa posição. Após destravar a balança, movimentaram-se os cursores até atingir o peso correto, travando-a novamente a seguir e procedendo à leitura. ${ }^{11}$

A estatura foi medida com o indivíduo de pé, na balança antropométrica, com a criança, ou adolescente, descalça e com a cabeça livre de adereços, no centro do equipamento. Mantiveram-se os indivíduos de pé, eretos, com os braços estendidos ao longo do corpo com a cabeça erguida, olhando para um ponto fixo na altura dos olhos, encostando os calcanhares, ombros e nádegas no antropômetro, unindo os pés, fazendo um ângulo reto com as pernas. Abaixou-se a parte móvel do equipamento, fixando-a contra a cabeça, com pressão suficiente para comprimir o cabelo. Realizou-se a leitura da estatura, sem soltar a parte móvel do equipamento. ${ }^{11}$

Para a técnica de aferição da PA foram adotadas as recomendações do National Hearth, Lung and Blood Institute ${ }^{12}$. Foi utilizado manguito de tamanho adequado, com largura em torno de $40 \%$ da circunferência do braço, medida à meia distância entre o acrômio e o olécrano e com bolsa de comprimento que envolveu completamente a circunferência do braço. Desse modo, na população do estudo, foram utilizados os manguitos de criança (largura: $9 \mathrm{~cm}$, comprimento: $18 \mathrm{~cm}$ ) e de adolescente (largura: $10 \mathrm{~cm}$, comprimento: $24 \mathrm{~cm}$ ) e estetoscópio infantil para aferição pelo método auscultatório, na artéria braquial direita.

As crianças permaneceram em repouso por 5 minutos, em ambiente calmo, sendo a aferição realizada com as mesmas sentadas, recostadas, com braço totalmente exposto e apoiado em suporte ao nível do coração. Foram utilizados esfignomanômetros aneroides, posicionados $2 \mathrm{~cm}$ acima da fossa antecubital direita. Insuflou-se a pera do aparelho até 20 mmHg após o desaparecimento do pulso braquial, procedendo- se, então, à liberação lenta da mesma, com ausculta dos sons. Para a determinação da PA sistólica foi considerado o primeiro som de Korotkoff e, para a determinação da PA diastólica, o quinto som (desaparecimento do ruído), conforme novos critérios do National Hearth,Lung and Blood Institute ${ }^{12}$. Ainda conforme esses novos critérios, foram realizadas três medidas, com intervalo de 1 a 2 minutos entre elas, sendo a média das duas últimas considerada a PA do escolar; caso as pressões sistólicas e/ou diastólicas obtidas apresentassem diferença maior que 4 $\mathrm{mmHg}$ entre elas, eram realizadas novas medidas, até que se obtivessem medidas com diferença inferior ou igual a $4 \mathrm{mmHg}$, utilizando-se a média das duas últimas medidas como a PA do escolar.

A partir da coleta dos dados, foi realizada a classificação das crianças e adolescentes em relação ao peso, a partir do Índice de Massa Corpórea (IMC), conforme novos critérios do Ministério da Saúde ${ }^{13} \mathrm{em}$ : IMC baixo (menor que percentil 3), IMC adequado (maior ou igual ao percentil 3 e menor que percentil 85), sobrepeso (maior ou igual ao percentil $85 \mathrm{e}$ menor que percentil 97) e obesidade (maior ou igual ao percentil 97).

Também foi realizada a classificação das crianças e adolescentes segundo a pressão arterial (PA). De acordo com as normas do National Hearth, Lung and Blood Institute (2005) ${ }^{12}$, também adotadas pela Sociedade Brasileira de Hipertensão Arterial, a classificação da PA será realizada a partir de uma tabela que correlaciona percentil de PA sistólica e diastólica com sexo, idade e percentil de estatura. Desse modo, foram consideradas: - Normotensas: as crianças e adolescentes com percentil abaixo de 90 , desde que inferiores a 120/80 mmHg;

- Limítrofes: as crianças e adolescentes com percentil maior ou igual a 90 e menor que 95 e crianças e adolescentes com PA maior ou igual a $120 / 80 \mathrm{mmHg}$;

- Hipertensas: as crianças e adolescentes com percentil igual ou superior a 95.

Ainda de acordo com tais diretrizes, ressaltamos que, quando as pressões sistólica e diastólica se situaram em categorias diferentes, a maior foi utilizada para a classificação da PA.

Após a categorização, os dados coletados foram digitados em planilha do programa Excel, transportados e analisados no programa estatístico Statistical Package for Social ScienceSPSS, versão 12, e apresentados por meio de tabelas e figuras.

Foram realizadas análises descritivas e de inferência. As análises descritivas corresponderam à análise da distribuição das frequências (absolutas e percentuais) e de medidas de tendência central (média). Para analisar a relação entre as variáveis foi realizado teste estatístico paramétrico (correlação de Pearson), de acordo com natureza da variável, sendo o nível de significância igual a 0,05. 


\section{RESULTADOS}

Foram estudados 148 crianças e adolescentes de uma escola pública de ensino fundamental. A distribuição das variáveis sociodemográficas e antropométricas na população estudada está apresentada na Tabela 1.

Tabela 1. Distribuição das variáveis de estudo. São José do Rio Preto, 2008.

\begin{tabular}{llcc}
\hline Variável & Masculino & $N$ & $\%$ \\
\hline Sexo & Feminino & 94 & 63,5 \\
& & 54 & 36,5 \\
Idade & 6 & 5 & 3,4 \\
& 7 & 34 & 23,0 \\
& 8 & 39 & 26,4 \\
& 9 & 35 & 23,6 \\
& 10 & 31 & 20,9 \\
& 11 & 4 & 2,7 \\
Classificação do IMC & & & 3,4 \\
& Baixo & 5 & 63,5 \\
& Adequado & 94 & 10,1 \\
& Sobrepeso & 15 & 23,0 \\
Classificação da PA & Obesidade & 34 & 85,8 \\
& Normotenso & 127 & 4,7 \\
& Limítrofe & 7 & 9,5 \\
\hline Total & Hipertenso & 14 & 100,0 \\
\hline
\end{tabular}

Os estudantes investigados eram em sua maioria do sexo masculino $(63,5 \%)$ e tinham em média 8,4 anos. A maior parte dos estudantes tinha IMC adequado - $63,5 \%$; entretanto, $36,5 \%$ dos estudantes apresentaram peso corporal alterado, sendo que $3,4 \%$ estavam abaixo do peso, $10,1 \%$ foram categorizados como com sobrepeso e $23 \%$ como obeso.

Comparando-se os resultados obtidos em relação ao IMC com outros estudos, realizados com escolares de faixa etária aproximada, nota-se o maior percentual de crianças obesas encontradas nesta pesquisa. Em estudo que avaliou o estado nutricional e a associação com o padrão de atividade física em escolares de 7 a 10 anos de idade (média de 8,8 anos), da Rede Municipal de Ensino de Corumbá (MS), encontrou-se uma prevalência de $12,7 \%$ de sobrepeso e de $9,4 \%$ de obesos entre a população estudada. ${ }^{14}$

Outra pesquisa conduzida em Feira de Santana (BA), com crianças de 5 a 9 anos (média de 7,1 anos) da rede pública de ensino, que objetivou identificar a influência de fatores biológicos e ambientais no desenvolvimento de $\mathrm{HA}$, demonstrou que $9,1 \%$ das crianças tinham sobrepeso e 4,3\%, obesidade. ${ }^{3}$ Um estudo internacional que teve o propósito de avaliar a prevalência de obesidade e comorbidades entre escolares de 6 a 13 anos em Taiwan indicou uma prevalência de 15\% de sobrepeso e $12 \%$ de obesidade. ${ }^{15}$

Com relação aos níveis de pressão arterial, aproximadamente $86 \%$ foram categorizados como normotensos, embora caiba ressaltar que $4,7 \%$ dos estudantes apresentaram níveis limítrofes de pressão arterial e 9,5\% foram categorizados como hipertensos.

A prevalência de HA na população de nosso estudo está em consonância com a variação nacional e internacional (entre 1 a $13 \%$ ), mas superior aos resultados obtidos em recentes estudos epidemiológicos brasileiros, que têm demonstrado prevalência entre $6 \%$ e $8 \%$. Deve-se considerar, ainda, que há também uma prevalência de níveis pressóricos limítrofes de 4,7\% entre a população do presente estudo; essa PA limítrofe, associada à presença de outros fatores de risco, pode levar a riscos cardiovasculares que variam de baixo a muito alto. ${ }^{12}$

Uma das possíveis causas dessa grande porcentagem de crianças com alterações de PA em nosso estudo poderia ser o fato de esses valores pressóricos terem sido obtidos em visita única.

Para caracterizar uma criança como portadora de HA é necessário que se obtenham três valores de PA alterados, em dias diferentes. ${ }^{12}$ Pesquisas longitudinais corroboram esse critério diagnóstico, demonstrando que altas prevalências de HA (até 13\%), identificadas no início dos estudos, tendiam a declinar consideravelmente em aferições subsequentes, chegando a $1 \%$ ou menos. ${ }^{16-18}$

Contudo, outra possível causa para a elevada prevalência de HA encontrada neste estudo diz respeito à correlação entre peso e pressão arterial, conforme explorado a seguir, a partir dos dados da Tabela 2. 
Tabela 2. Correlação da classificação do índice de massa corporal e a classificação da pressão arterial da população pesquisada. São José do Rio Preto - 2008

\begin{tabular}{|c|c|c|c|c|c|c|c|c|c|c|c|}
\hline & \multicolumn{11}{|c|}{ Classificação do IMC } \\
\hline & & \multicolumn{2}{|c|}{ Baixo } & \multicolumn{2}{|c|}{ Adequado } & \multicolumn{2}{|c|}{ Sobrepeso } & \multicolumn{2}{|c|}{ Obesidade } & \multicolumn{2}{|c|}{ Total } \\
\hline & & $\mathrm{N}$ & $\%$ & $N$ & $\%$ & $N$ & $\%$ & $N$ & $\%$ & $N$ & $\%$ \\
\hline \multirow{3}{*}{$\begin{array}{l}\text { Classifica ção da } \\
\text { PA }\end{array}$} & Normotenso & 5 & 100,0 & 82 & 87,2 & 12 & 80,0 & 28 & 82,4 & 127 & 85,8 \\
\hline & Limítrofe & - & - & 4 & 4,3 & - & - & 3 & 8,8 & 7 & 4,7 \\
\hline & Hipertenso & - & - & 8 & 8,5 & 3 & 20,0 & 3 & 8,8 & 14 & 9,5 \\
\hline Total & & 5 & 100,0 & 94 & 100,0 & 15 & 100,0 & 34 & 100,0 & 148 & 100,0 \\
\hline
\end{tabular}

Ao se analisar os níveis pressóricos de acordo com a classificação do IMC como peso adequado e sobrepeso/ obesidade, apresentados na Tabela 2, verificou-se que mais de $87 \%$ das crianças e adolescentes que tinham IMC classificado como adequado eram normotensos, embora 4,3\% e 8,5\% tenham apresentado níveis limítrofes e hipertensos, respectivamente.

Dentre os estudantes que apresentaram índice de sobrepeso, $80 \%$ eram normotensos e $20 \%$, hipertensos. De modo semelhante, aproximadamente $82 \%$ das crianças e adolescentes obesas eram normotensos; entretanto entre estes, $8,8 \%$ foram classificados como limítrofes e 8,8, como hipertensos.

A prevalência de HA em escolares com estado nutricional normal foi muito próxima à obtida por um estudo epidemiológico que avaliou alunos entre 7 e 17 anos de escolas públicas e privadas de Maceió. Embora os critérios para classificação do IMC utilizados diferissem daqueles utilizados pelo nosso estudo, encontrou-se que a HA esteve presente em $8,1 \%$ dos indivíduos com estado nutricional normal (IMC superior ao percentil $5 \mathrm{e}$ inferior ao percentil 85), em 12,1\% dos indivíduos com risco de sobrepeso (IMC igual ou superior ao percentil 85 , aproximadamente o que consideramos sobrepeso) e em $28,6 \%$ dos indivíduos com sobrepeso (IMC igual ou superior ao percentil 95, aproximadamente o que consideramos obesidade). ${ }^{6}$

De maneira semelhante, outros estudos apontam maior prevalência de HA entre crianças e adolescentes obesos ou com sobrepeso do que naqueles com estado nutricional adequado, em consonância com os resultados obtidos neste estudo. Contudo, em tais estudos há uma maior prevalência de HA entre indivíduos com obesidade do que entre aqueles com sobrepeso $0^{6,3,15}$ ao contrário do que foi verificado em nosso estudo. Essa diferença poderia ser atribuída ao fato de que tais autores utilizaram um critério diferente para a classificação do estado nutricional, considerando as categorias sobrepeso e obesidade como IMC igual ou superior ao percentil 85 e 95 para idade e sexo, respectivamente. Já a classificação utilizada neste estudo considera os percentis de corte como 85 e 97, ampliando, desse modo, o número de crianças classificadas como com sobrepeso e diminuindo os obesos, em comparação à classificação anterior. Desse modo, é possível que algumas crianças e adolescentes hipertensos, classificados atualmente como com sobrepeso, teriam sido classificados como obesos se utilizados os critérios anteriores.

As variáveis também foram analisadas em sua forma não categorizadas por meio do teste de correlação de Pearson, apresentado na Figura 1. Como era de se esperar, as variáveis peso e IMC apresentaram correlação forte e positiva $(r=0,916$, $p<0,01)$. 0 mesmo ocorreu entre as variáveis PAS e PAD $(r=0,648, p<0,01)$. Peso e PAS apresentaram correlação forte e positiva ( $r=0,429, p<0,01)$, enquanto o peso e a PAD apresentaram correlação moderada e positiva $(r=0,172$, $p<0,05)$. 0 IMC apresentou correlação forte e positiva com a PAS $(r=0,417, p<0,01)$ e moderada e positiva com a PAD $(r=0,175, p<0,05)$.

rigura I. Correlação de Pearson para va riáveis quantitativas.

\begin{tabular}{l|c|c|c|c|}
\hline & Peso & IMC & PAS & PAD \\
\hline Peso & 1 & & & \\
\hline IMC & $0,916^{* *}$ & 1 & & \\
\hline PAS & $0,429^{* *}$ & $0,417^{* *}$ & 1 & \\
\hline PAD & $0,172^{*}$ & $0,175^{*}$ & $0,648^{* *}$ & 1 \\
\hline
\end{tabular}
** Correlação significativa com $p=0,01$.
${ }^{*}$ Correlação significativa com $p=0,05$.


Correlação pressão arterial e peso em crianças Zanoti MDU, Pina JC, Manetti ML

Ao se analisar os resultados obtidos no teste de correlação, verificou-se a existência de correlação positiva e, de modo geral, forte, entre o peso e o IMC e a pressão arterial na população investigada, indicando que quanto maior o peso/IMC maiores serão os níveis pressóricos.

Uma associação significativa foi identificada entre HA e sobrepeso $(p=0,04)$ e obesidade $(p=0,000)$ em uma amostra de crianças da rede de ensino público e privado da zona urbana de Feira de Santana, BA. A razão de chance calculada pela pesquisa mostrou associação 13 vezes maior entre crianças portadoras de obesidade e desenvolvimento de HA. ${ }^{3}$

Nesse contexto, a prevalência de HA encontrada na população de nosso estudo, superior à variação nacional, poderia também ser explicada pela alta prevalência de obesidade e sobrepeso entre essas crianças e adolescentes. Contudo, fazse necessário um acompanhamento dessa população, realizando novas aferições em ocasiões distintas, a fim de atender aos critérios diagnósticos preconizados atualmente.

\section{CONCLUSÃO}

0 presente estudo identificou uma prevalência de HA de 9,5\% entre crianças e adolescentes de uma escola da rede pública do interior paulista. Identificou, ainda, uma grande porcentagem de indivíduos obesos e com sobrepeso (23\% e
$10,1 \%$, respectivamente) e a existência de correlação positiva e, de modo geral, forte, entre o peso / IMC e a pressão arterial na população investigada.

No entanto, há de se considerar que os níveis pressóricos foram obtidos em somente uma oportunidade. Portanto, a população deverá ser reavaliada em mais duas ocasiões para se definir a presença ou não de HA. Se confirmada a classificação, tais escolares serão encaminhados ao serviço de saúde para investigação e acompanhamento.

Nesse sentido, este estudo insere-se no contexto das diretrizes governamentais de integração setorial para atender às necessidades de saúde da população, à medida que desenvolve a integração entre instituições de ensino e de saúde. Seus resultados também contribuem para o diagnóstico precoce de alterações de PA na população estudada, além da prevenção deste distúrbio nos obesos e com sobrepeso, que envolve 0 esclarecimento de educadores e familiares sobre a importância de mudanças no estilo de vida.

Contudo, diante da ausência de estudos publicados que utilizem a nova classificação de IMC para crianças e adolescentes, faz-se necessário dar continuidade aos estudos, em um número maior dessa população, assim como os serviços de saúde devem realizar aferição de pressão arterial nos atendimentos de rotina à criança e ao adolescente.

\section{REFERÊNCIAS}

1. Rhein SO. Hipertensão arterial sistêmica, medida de circunferência da cintura e consumo alimentar em adolescentes com diagnóstico antropométrico de obesidade [tese de doutorado]. São Paulo (SP): Escola Paulista de Medicina/USP; 2005

2. Novaes JF, Franceschini SCC, Priore SE. Obesidade infantil: um distúrbio nutricional em ascensão no mundo moderno. Nutrire Rev Soc Bras Aliment Nutr 2007 abr; 32(1): 59-75.

3. Oliveira AMA, Oliveira AC, Almeida MS, Almeida FS, Ferreira, JBC, Silva CEP, et al. Fatores ambientais e antropométricos associados à hipertensão arterial infantil. Arq Bras Endocrinol Metab 2004 dez; 48(6): 849-54.

4. Moreira RP, Guedes NG; Cavalcante TF, Silva VM, Araujo TL. Oficinas educativas sobre hipertensão arterial: um estudo quase-experimental sobre avaliação da eficácia. Online Braz J Nurs [periódico na internet]. 2005 dez; [citado 04 fev 2008]; 4(3): [aprox. 13 telas]. Disponível em: http://www.uff.br/objnursing/index.php/nursing/article/view/10/ 5.

5. Barreira AK, Couto GBL, Vasconcelos MMVB, Vianna RBC. Hipertensão arterial na infância. JBP: $\mathrm{j}$ bras odontopediatr \&odontol bebe 2003 mar/abr; 6(30): 131-36.

6. Moura AA, Silva MAM, Ferraz MRMT, Rivera IR. Prevalência de pressão arterial elevada em escolares de Maceió. J Pediatr 2004; 80(1): 35-40.

7. Salgado CM, Carvalhaes JTA. Hipertensão arterial na infância. J Pediatr 2003; 79(S1): 115-24.
8. Alves JGB, Montenegro FMU, Oliveira FA, Alves RV. Prática de esportes durante a adolescência e atividade física de lazer na vida adulta. Rev Bras Med Esporte 2005 set/out; 11(5): 291-94.

9. Vasconcelos VL, Lapa TM, Carvalho EF. Prevalência de sobrepeso e obesidade em adolescentes masculinos nas macrorregiões do Brasil, 1980-2000. Esc Anna Nery Rev Enferm 2006; 10(3): 417-24.

10. Rêgo ALV, Chiara VL. Nutrição e excesso de massa corporal: fatores de risco cardiovascular em adolescentes. Rev Nutr 2006 nov/ dez; 19(6): 705-12.

11. Ministério da Saúde (BR). Vigilância Alimentar e Nutricional-SISVAN. Orientações básicas para a coleta, o processamento, a análise de dados e a informação em serviços de saúde. Brasília( DF); 2004.

12. National Institute of Health (USA) National Heart, Lung and Blood Institute. Department of Health and Human Services. The four th report on the diagnosis, evaluation and treatment of high blood pressure in children and adolescents. NIH Publ; 2005.

13. Ministério da Saúde (BR). Secretaria de Atenção à Saúde. Departamento de Atenção Básica. Coordenação Geral da Política de Alimentação e Nutrição. Orientações para a coleta e análise de dados antropométricos em serviços de saúde. Material Preliminar da Norma Técnica-SISVAN. Brasília (DF); 2008.

14. Baruki SBS, Rosado LEFPL, Rosado GP, Ribeiro RCL. Associação entre estado nutricional e atividade física em escolares da Rede 
Municipal de Ensino em Corumbá - MS. Rev Bras Med Esporte 2006 mar/abr;12(2): 90-4.

15. Chu NF, Pan WH. Prevalence of obesity and its comorbidities among schoolchildren in Taiwan. Asia Pac J Clin Nutr. 2007; 16(S2):6017.

16. Clarke WR, Woolson RF, Lauer RM. Changes in ponderosity and blood pressure in childhood: the muscatine study. Am J Epidemiol 1986; 124:195-206.
17. Londe S. Blood pressure in children as determined under office condition. Clin Pediatr 1966; 5: 71-8.

18. Adrogué HE, Sinaiko AR. Prevalence of hypertension in junior high school-aged children: effect of new recommendations in the 1996. Updated Task Force Report. Am J Hypertens 2001; 14: 412-14. 